\title{
NONLINEAR RESONANCE IN SYSTEMS OF CONSERVATION LAWS*
}

\author{
ELI ISAACSON $\dagger$ AND BLAKE TEMPLE $\ddagger$
}

\begin{abstract}
The Riemann problem for a general inhomogeneous system of conservation laws is solved in a neighborhood of a state at which one of the nonlinear waves in the problem takes on a zero speed. The inhomogeneity is modeled by a linearly degenerate field. The solution of the Riemann problem determines the nature of wave interactions, and thus the Riemann problem serves as a canonical form for nonlinear systems of conservation laws. Generic conditions on the fluxes are stated and it is proved that under these conditions, the solution of the Riemann problem exists, is unique, and has a fixed structure; this demonstrates that, in the above sense, resonant inhomogeneous systems generically have the same canonical form. The wave curves for these systems are only Lipschitz continuous in a neighborhood of the states where the wave speeds coincide, and so, in contrast to strictly hyperbolic systems, the implicit function theorem cannot be applied directly to obtain existence and uniqueness. Here we show that existence and uniqueness for the Riemann problem is a consequence of the uniqueness of intersection points of Lipschitz continuous manifolds of complementary dimensions. These systems are resonant for two reasons: The linearized problem exhibits classical resonant behavior, while the nonlinear initial value problem exhibits a "nonlinear resonance" in the sense that wave speeds from different families of waves are not distinct; so the number of times a pair of waves can interact in a given solution cannot be bounded a priori. Since waves are reflected in other families every time a pair of waves interact, a proliferation of reflected waves can occur by the interaction of a single pair of waves. Examples of resonant inhomogeneous systems are observed in model problems for the flow of a gas in a variable area duct and in Buckley-Leverett systems that model multiphase flow in a porous medium.
\end{abstract}

Key words. Riemann problem, nonstrictly hyperbolic, resonance

AMS(MOS) subject classifications. 35L65, 35L67, 65M10, 76N99

1. Introduction. We are interested in characterizing the resonant behavior that occurs in an arbitrary inhomogeneous system of conservation laws in a neighborhood of a state at which one of the nonlinear wave families has a zero wave speed. By an inhomogeneous system of conservation laws, we mean a system of the form

$$
u_{t}+f(a, u)_{x}=0 \text {, }
$$

where $a=a(x)$ is a variable function of $x$ alone; thus $a$ represents an inhomogeneity in the problem. We express this by the additional conservation law

$$
a_{t}=0 \text {. }
$$

(Systems of this form were previously identified by the authors when we outlined a program for classifying the solutions of nonstrictly hyperbolic systems (cf. [5], [6], [8]).) Our general problem thus becomes

$$
U_{t}+F(U)_{x}=0
$$

where $U=(a, u), F(U)=(0, f(a, u)), u=\left(u_{1}, u_{2}, \cdots, u_{n}\right) \in R^{n}, f=\left(f_{1}, f_{2}, \cdots, f_{n}\right) \in$ $R^{n}, x \in R, t \geqq 0$. System (1.3) is a system of $n+1$ equations in the $n+1$ unknowns $a$, $u_{1}, \cdots, u_{n}$. We assume that, for each value of $a$, system (1.1) is a strictly hyperbolic system of $n$ equations, and that each of the characteristic fields is either genuinely nonlinear or linearly degenerate [3], [10], [14]. Equation (1.2) produces a linearly

* Received by the editors August 24, 1990; accepted for publication (in revised form) January 31, 1991. This research was partially supported by National Science Foundation grant DMS-86-13450 and by the Institute of Theoretical Dynamics, University of California at Davis, Davis, California 95616.

$\dagger$ Department of Mathematics, University of Wyoming, Laramie, Wyoming 82071.

¥Department of Mathematics, University of California at Davis, Davis, California 95616. 
degenerate field (i.e., $\nabla \lambda_{0} \cdot \mathbf{R}_{0}=0$ ) in system (1.3) with eigenvalue $\lambda_{0}=0$ and corresponding eigenvector $\mathbf{R}_{0}$ of the Jacobian matrix $\partial F / \partial U$. The remaining eigenvalues $\lambda_{1}<\lambda_{2}<\cdots<\lambda_{n}$ of system (1.3) correspond to the eigenvalues of system (1.1) and have corresponding right eigenvectors $\mathbf{R}_{1}=\left(0, \mathbf{r}_{1}\right), \cdots, \mathbf{R}_{n}=\left(0, \mathbf{r}_{n}\right)$, which lie in the hyperplane $a=$ const. Here the vectors $\mathbf{r}_{1}, \cdots, \mathbf{r}_{n} \in R^{n}$ at a state $U=(a, u)$ are the unit right eigenvectors of the corresponding $n \times n$ matrix $\partial f / \partial u$. We let $\mathbf{l}_{1}, \cdots, \mathbf{l}_{\mathbf{n}}$ denote the corresponding left eigenvectors of the matrix $\partial f / \partial u$ normalized so that $\mathbf{l}_{j} \cdot \mathbf{r}_{j}=1$ for $j=1, \cdots, n$. We wish to study system (1.3) in a neighborhood of a state $U_{*}=$ $\left(a_{*}, u_{*}\right)$ at which a nonlinear family of waves in system (1.3) has a zero wave speed. Thus we assume that

$$
\lambda_{k}\left(U_{*}\right)=\lambda_{0}=0
$$

and that

$$
\left.\nabla \lambda_{k} \cdot \mathbf{R}_{k}\right|_{U_{*}} \neq 0
$$

In Theorem 3.1 we show that these assumptions, together with the nondegeneracy assumption

$$
\left.\mathbf{l}_{k} \cdot f_{a}\right|_{U_{*}} \neq 0
$$

guarantee that, in a neighborhood of the state $U_{*}$, the Riemann problem has a unique solution with a canonical structure. (Here $f_{a}$ denotes the partial derivative $\partial f / \partial a$.) The Riemann problem for (1.3) is the following initial value problem for piecewise constant initial data:

$$
U(x, 0)= \begin{cases}U_{L} & \text { for } x<0 \\ U_{R} & \text { for } x>0\end{cases}
$$

The Riemann problem is fundamental to the study of (1.3) because it identifies the elementary waves that propagate-typically, shock waves, rarefaction waves, and contact discontinuities. Our main result is that, for each pair of states $U_{L}$ and $U_{R}$ in a neighborhood of $U_{*}$, there is a unique solution of the Riemann problem that is determined by a canonical underlying structure of the elementary waves in the problem.

One consequence of the genericity assumptions (1.5) and (1.6) is that the linearized system in the $0-\lambda_{k}$ block has the normal form

$$
\left[\begin{array}{l}
a \\
u
\end{array}\right]_{t}+\left[\begin{array}{ll}
0 & 0 \\
1 & 0
\end{array}\right]\left[\begin{array}{l}
a \\
u
\end{array}\right]_{x}=0
$$

Resonant behavior occurs in the linearized problem because the solution $u(x, t)=$ $a^{\prime}(x) t+c$ blows up as $t$ tends to infinity. The nonlinear initial value problem exhibits a "nonlinear resonance" in the sense that wave speeds from different families of waves are not distinct, and so the number of times a pair of waves can interact in a given solution cannot be bounded a priori. Consequently, since waves are reflected in other families every time a pair of waves interact, a proliferation of reflected waves can be produced by the interaction of a single pair of waves. Another consequence of (1.4)(1.6) is that there is a surface on which wave speeds coincide, and thus our systems do not fit the framework of [15] in which the coincidence occurs at a single "umbilic" point.

Special cases of (1.3) are observed in model problems for the flow of a gas in a variable area duct and in Buckley-Leverett systems that model multiphase flow in a porous medium. In the latter case, the model equations do not have the form of an inhomogeneous system of conservation laws, but there is a Lagrangian transformation that maps the model equations to an equivalent system (in the weak sense) that does 
have this form. (The transformation was shown to the authors by Marchesin and Patino.) Examples of such systems have been studied by Keyfitz and Kranzer [9] and by the authors [4], [17]. Under the Lagrangian transformation, these are equivalent to system (1.3), where $u$ is a scalar. We refer to this as the scalar case. In each of these examples, a coincidence of wave speeds occurs, and (1.4)-(1.6) are satisfied. Many, but not all, of the features in the scalar case carry over to the case where $u$ is a vector and where (1.1) describes an inhomogeneous system of conservation laws. For example, there are, in general, $n+2$ waves in the solution of an inhomogeneous system, even though there are only $n+1$ equations, and Riemann problem solutions depend continuously on the data in $x, t$-space, but not in state space. However, unlike the scalar case, the wave curves for systems are only Lipschitz continuous curves near the point of resonance. This makes direct application of the implicit function theorem difficult, and we show that the existence and uniqueness of solutions of the Riemann problem in a neighborhood of a point of resonance is a consequence of the uniqueness of intersection points of Lipschitz continuous manifolds having complementary dimensions.

Wave interactions are significantly more complicated in system (1.3) than in a strictly hyperbolic system. For example, Temple showed in [17] that solutions satisfy a time-independent estimate on the total variation as measured under a singular transformation of the conserved quantities. Such time-independent estimates are relevant to the study of the asymptotic decay of solutions into noninteracting wave patterns as $t$ tends to infinity. This was analyzed in [7], where it was shown that the decreasing nonlinear functional introduced in [17] is minimized on a unique set of noninteracting waves that, in general, are inadmissible solutions of the Riemann problem; it was conjectured that these are the time-asymptotic waves in the solution. In one dimension, the total variation of a solution at time $t$ is the most natural measure of the total strength of the waves in the solution at time $t$, and thus it is natural to expect that the total variation of the solution at time $t$ should be bounded by the total variation at time $t=0$, at least for sufficiently weak waves. This time-independent estimate was proved by Glimm for strictly hyperbolic systems in his fundamental paper [2] and has been applied to obtain rates of decay of the solutions asymptotically as $t$ tends to infinity. Simple examples, however, show that the total variation of solutions of (1.3) at a time $t>0$ cannot be bounded by the total variation at time $t=0$ in the space of conserved quantities, uniformly in time, even when $u$ is a scalar and $a(x)$ is smooth. The analysis in [17] gives time-independent bounds on solutions when $u$ is a scalar and is based on a singular transformation of the $(a, u)$-plane. We believe that an analysis of the elementary waves through the theory of the Riemann problem may well be useful for obtaining time-independent bounds on solutions and a corresponding understanding of the time-asymptotic decay of solutions for vector-valued $u$ because the elementary waves in the solutions of the Riemann problem describe the timeasymptotic wave patterns to which an arbitrary solution evolves. To extend the results in the scalar problem to the case when $u$ is a vector, a sharper bound on the total variation of solutions is needed, as well as a quadratic potential interaction functional. Such a quadratic function has not been found in any other case in which there is no a priori bound on the number of times a pair of waves can interact.

In $\S 2$ we describe two physical problems that are modeled by resonant inhomogeneous systems. In $\S 3$ we state and discuss Theorem 3.1, which is the main result of the paper. We introduce Lipschitz continuous manifolds with approximate tangent vectors in $\S 3$, and we show in Theorem 4.4 that two such manifolds having complementary dimensions intersect in a unique point. In $\S 5$ we prove Theorem 3.1 by constructing Lipschitz continuous manifolds from wave curves and applying the 
results of $\S 4$. Our work on resonant inhomogeneous systems was influenced significantly by Marchesin and Paes-Leme [13].

2. Applications. In this section we describe two physical settings in which resonance in inhomogeneous systems of conservation laws arises.

Flow in a variable area duct. The equations for the flow of a gas in a variable area duct with cross-sectional area $a(x)$ are [1]

$$
\begin{gathered}
\rho_{t}+(\rho u)_{x}=-\left(a^{\prime} / a\right) \rho u \\
(\rho u)_{t}+\left(\rho u^{2}+p\right)_{x}=-\left(a^{\prime} / a\right) \rho u^{2} \\
E_{t}+[(E+p) u]_{x}=-\left(a^{\prime} / a\right)[(E+p) u] .
\end{gathered}
$$

The equations express the conservation of mass $(\rho)$, momentum $(\rho u)$, and energy $(E)$, respectively. We say that resonance occurs in transonic flow because one of the nonlinear waves can have a zero wave speed (cf. Liu [11]). Liu was the first to study the initial value problem for these equations using Glimm's random choice method [2], and he proved convergence of the method for solutions taking values in a neighborhood of a state $(\rho, \rho u, E)$ at which the wave speeds are bounded away from zero (see [11] and the references therein). In [12] Liu gives a fairly complete analysis of a nonconservative scalar model for (2.1) in which resonance occurs. At present, however, there is no general proof that Glimm's method converges for systems in the transonic regime.

To obtain a model problem, we rewrite system (2.1) in the form

$$
\begin{aligned}
(a \rho)_{t}+(a \rho u)_{x} & =0, \\
(a \rho u)_{t}+\left(a \rho u^{2}+a p\right)_{x} & =a^{\prime} p, \\
(a E)_{t}+[a(E+p) u]_{x} & =0,
\end{aligned}
$$

with the supplementary equation

$$
a_{t}=0 \text {. }
$$

Dropping the zero-order term from the right side of (2.2) yields a mathematical model for the resonant behavior that occurs in transonic flow. The resulting system has the form of system (1.3). Note that this reduced system also can be viewed as the first system to be solved in a numerical time-splitting method for (2.2).

In the special case where $p=c^{2} \rho$ (isothermal flow), the energy equation drops out, and the zero-order term can be incorporated into the fluxes to obtain the system

$$
\begin{aligned}
a_{t} & =0, \\
(a \rho)_{t}+(a \rho u)_{x} & =0, \\
u_{t}+\left(u^{2} / 2+c^{2} \log \rho\right)_{x} & =0 .
\end{aligned}
$$

Although this does not supply a physical conservation form for the original problem, it does provide a mathematical model containing a similar nonlinear resonance in the transonic regime. A straightforward calculation verifies that $\left.\mathbf{l}_{k} \cdot f_{a}\right|_{U_{*}}=-c^{2} \rho$, and thus conditions (1.4)-(1.6) are valid for system (2.4). For flow in a variable area duct, we believe that these models isolate an important component in the complicated behavior of transonic flow. Marchesin and Paes-Leme [13] study this system in an analysis of the Riemann problem obtained by taking $a$ to be piecewise constant; our point of view was influenced significantly by their analysis. 
Buckley-Leverett systems. We call the following equations polymer equations because they arise as a model for the polymer flooding of an oil reservoir (i.e., two-phase, three-component flow in a porous medium [4], [17]):

$$
\begin{aligned}
s_{t}+f(s, c)_{x} & =0, \\
(c s)_{t}+(c f(s, c))_{x} & =0 .
\end{aligned}
$$

Here $s$ and $c$ denote the water saturation and the polymer concentration, respectively, and satisfy $0<s \leqq 1$ and $0 \leqq c \leqq 1$, while $f=f(s, c)$ is a constitutive relation. The structure of solutions is determined by qualitative properties of $f$ [4], [17]. The eigenvalues of system (2.5) coincide when $f_{s}=f / s$. The Riemann problem for this system is studied by Isaacson in [4], while Keyfitz and Kranzer [9] study the Riemann problem for the equivalent system

$$
\begin{gathered}
u_{t}+[u g(u, v)]_{x}=0, \\
v_{t}+[v g(u, v)]_{x}=0,
\end{gathered}
$$

which arises in their study of elasticity. The polymer interpretation of these equations suggests a natural Lagrangian transformation of the variables. In this model, $g=f / s$ is the particle velocity of the water, and so the trajectories of the water particles are given by solutions of the ordinary differential equation $x^{\prime}=g(s(x, t), c(x, t))$. We can thus define a solution-dependent mapping of the independent variables $(x, t)$ to $(\xi, t)$ so that $\xi=$ const defines the particle trajectories in the transformed, or Lagrangian, coordinates $(\xi, t)$. This transformation is defined by

$$
\xi(x, t)=\int_{x(0, t)}^{x} s(z, t) d z,
$$

where $x(0, \cdot)$ is the particle path through the point $x=0$ at time $t=0$. Rewriting system (2.5) with respect to $(\xi, t)$ yields the equivalent system

$$
\begin{aligned}
c_{t} & =0, \\
(1 / s)_{t}-g(s, c)_{\xi} & =0,
\end{aligned}
$$

which has the form (1.1), (1.2) when we make the identifications $u=1 / s, a=c$, and $h=-g$ (cf. [1, p. 30]). Systems (2.5) and (2.8) are equivalent in the sense that they determine the same shock wave solutions under the 1-1 mapping given by the Lagrangian change of variables. In the case where the nonlinear family of waves is genuinely nonlinear on the transition surface [4], [17], system (2.8) satisfies assumptions (1.4)-(1.6) at points where $\lambda_{0}=\lambda_{1}$.

3. The Riemann problem. We consider the system of equations $a_{t}=0, u_{t}+$ $f(a, u)_{x}=0$, where $u=\left(u_{1}, u_{2}, \cdots, u_{n}\right) \in R^{n}$ and $a \in R$. We can write this system in the form (1.3) by taking $U=(a, u)$ and $F=(0, f)$. Here $a=a(x)$ is an inhomogeneity in the equations, and $a_{t}=0$ gives rise to a linearly degenerate field with wave speed $\lambda_{0}=0$. We consider the Riemann problem for weak solutions in a neighborhood of a state $U_{*}=\left(a_{*}, u_{*}\right)$ at which

$$
\lambda_{1}<\cdots<\lambda_{k}=\lambda_{0}<\cdots<\lambda_{n} .
$$

This represents the simplest example of a coincidence of wave speeds. Our main result is the following theorem. 
THeOREM 3.1. Assume that, in a neighborhood of a state $U_{*}=\left(a_{*}, u_{*}\right)$, the $n \times n$ system

$$
u_{t}+f(a, u)_{x}=0,
$$

is strictly hyperbolic for each fixed value of $a$, and is either genuinely nonlinear or linearly degenerate in each characteristic field. Let

$$
\lambda_{1}(a, u)<\lambda_{2}(a, u)<\cdots<\lambda_{n}(a, u)
$$

denote the eigenvalues of this system with corresponding right eigenvectors $\mathbf{r}_{1}, \cdots, \mathbf{r}_{\mathbf{n}}$ and left eigenvectors $\mathbf{l}_{1}, \cdots, \mathbf{l}_{n}$. Assume that the function $f$ satisfies the following conditions at the state $U_{*}$ :

(i) $\lambda_{k}\left(a_{*}, u_{*}\right)=0$,

(ii) $\left.\nabla \lambda_{k} \cdot \mathbf{r}_{k}\right|_{U_{*}} \neq 0$,

(iii) $\left.\mathbf{l}_{k} \cdot f_{a}\right|_{U_{*}} \neq 0$.

Then there exists a unique solution of the Riemann problem in a neighborhood of $U_{*}$, and this solution depends continuously in physical space on the left and right states. Moreover, for every $f$ in this class, the solutions exhibit the same qualitative behavior.

We discuss the main consequences of assumptions (i)-(iii) here and complete the proof of Theorem 3.1 in the following two sections. First, assumption (ii) guarantees that the equation $\lambda_{k}=0$ defines a smooth $n$-dimensional surface locally in $R^{n+1}$ that passes through the state $U_{*}$. We call this the transition surface $\mathfrak{T}$. The eigenvector $\mathbf{R}_{k}=\left(0, \mathbf{r}_{k}\right)$ points along the hyperplane $a=$ const, and condition (ii) guarantees that the integral curves of $\mathbf{R}_{k}$ cut the transition surface $\mathfrak{I}$ transversally. Moreover, condition (iii) implies that the $n \times(n+1)$ matrix $\partial f / \partial U=\left[f_{a} ; f_{u}\right]$ has maximal rank $n$ at $U_{*}$. To see this, note that, since $\lambda_{k}=\lambda_{0}=0$ and $\lambda_{i} \neq \lambda_{k}$ at $U_{*}, f_{u}$ has rank $n-1$. Moreover, since $\left.\mathbf{l}_{k} \cdot f_{a}\right|_{U_{*}} \neq 0$, and since $\mathbf{l}_{k} \cdot \mathbf{r}_{i}=0$ for $i \neq k, \partial f / \partial U$ must have rank $n$. Consequently, the Jacobian matrix $d F \equiv \partial F / \partial U$ has rank $n$ at $U_{*}$ since $F=(0, f)$, so that $\partial F / \partial U$ has the Jordan normal form

$$
\left[\begin{array}{llllll}
\lambda_{1} & & & & & \\
& \lambda_{k-1} & & & & \\
& & 0 & 1 & & \\
& & 0 & 0 & & \\
& & & & \lambda_{k+1} & \\
& & & & & \lambda_{n}
\end{array}\right]
$$

at $U_{*}$. We conclude that $\partial F / \partial U$ has the normal form $(J)$ for every $U \in \mathfrak{T}$ in a neighborhood of $U_{*}$ because (iii) is an open condition. In particular, this implies that the eigenvectors $\mathbf{R}_{0}$ and $\mathbf{R}_{k}$ can be chosen off $\mathfrak{I}$ to have smooth extensions that agree on $\mathfrak{I}$. Thus, by condition (ii), the integral curves for both $\mathbf{R}_{0}$ and $\mathbf{R}_{k}$ cut the surface $\mathfrak{I}$ transversally near the state $U_{*}$. Specifically, off the surface $\mathfrak{I}, \mathbf{R}_{0}=$ $c\left(1,-(\partial f / \partial u)^{-1}(\partial f / \partial a)\right)$, where $c$ is chosen to make $\mathbf{R}_{0}$ a unit vector, so that $\mathbf{R}_{0}$ continues smoothly to $\mathbf{R}_{k}=\left(0, \mathbf{r}_{k}\right)$ on $\mathfrak{T}$. Finally, condition (ii) implies that the integral curves of $\mathbf{R}_{0}$ that pass through states $U_{0} \in \mathfrak{I}$ in a neighborhood of $U_{*}$ do not cross the hyperplane $a=a_{0}$ at $U_{0}$, and this implies that the integral curves of $\mathbf{R}_{0}$ passing through states $U_{0} \in \mathfrak{I}$ must cross the hyperplane $a=$ const exactly twice at values of $a$ on one side of $a=a_{0}$. This follows directly from the following lemma.

LEMMA 3.2. Let $U(\varepsilon)=(a(\varepsilon), u(\varepsilon))$ denote the arclength parameterization of the integral curve of $\boldsymbol{R}_{0}=\left(a_{0}, \mathbf{r}_{0}\right)$ that satisfies $U(0)=U_{*}$, and assume conditions (i)-(iii) 
of Theorem 3.1. Then

$$
a^{\prime}(0)=0 \text { and } a^{\prime \prime}(0)=\left.\nabla a_{0} \cdot \mathbf{R}_{0}\right|_{U_{*}}=-\frac{\nabla \lambda_{k} \cdot \mathbf{r}_{k}}{\mathbf{l}_{k} \cdot f_{a}} \neq 0 .
$$

Proof. The specified integral curve is defined by $U^{\prime}=\mathbf{R}_{0}(U)$ with $U(0)=U_{*}$. That is, $a^{\prime}=a_{0}$ and $u^{\prime}=\mathbf{r}_{0}$ with $a(0)=a_{*}$ and $u(0)=u_{*}$. In particular, $a^{\prime}(0)=0$, since $\mathbf{R}_{0}=\mathbf{R}_{k}=\left(0, \mathbf{r}_{k}\right)$ on $\mathfrak{T}$. In addition, the integral curve satisfies

$$
f(a(\varepsilon), u(\varepsilon))=f\left(a_{*}, u_{*}\right)
$$

Differentiating (3.2) with respect to $\varepsilon$ yields

$$
f_{a} a^{\prime}+f_{u} u^{\prime}=0
$$

We write

$$
u^{\prime}=\sum_{i=1}^{n} c_{i}(\varepsilon) \mathbf{r}_{i}(\varepsilon)
$$

where $\mathbf{r}_{i}(\varepsilon)=\mathbf{r}_{i}(U(\varepsilon))$ is the $i$ th right eigenvector of $f_{u}$ at $U(\varepsilon)$. Since $u^{\prime}(0)=\mathbf{r}_{0}=\mathbf{r}_{k}$, we must have that $c_{i}(0)=0$ for $i \neq k$, and $c_{k}(0)=1$. Differentiating (3.3) with respect to $\varepsilon$, we obtain that

$$
f_{a a}\left(a^{\prime}\right)^{2}+f_{a} a^{\prime \prime}+\frac{d}{d \varepsilon}\left\{f_{u} u^{\prime}\right\}=0
$$

However,

$$
f_{u} u^{\prime}=f_{u} \sum_{i=1}^{n} c_{i}(\varepsilon) \mathbf{r}_{i}(\varepsilon)=\sum_{i=1}^{n} c_{i}(\varepsilon) \lambda_{i}(\varepsilon) \mathbf{r}_{i}(\varepsilon)
$$

and thus

$$
\frac{d}{d \varepsilon}\left\{f_{u} u^{\prime}\right\}=\sum_{i=1}^{n} c_{i}^{\prime}(\varepsilon) \lambda_{i}(\varepsilon) \mathbf{r}_{i}(\varepsilon)+\sum_{i=1}^{n} c_{i}(\varepsilon) \frac{d}{d \varepsilon}\left\{\lambda_{i}(\varepsilon) \mathbf{r}_{i}(\varepsilon)\right\}
$$

At $\varepsilon=0$, we have that $\lambda_{k}(0)=0$ and $a^{\prime}(0)=0$, so that

$$
\sum_{i=1}^{n} c_{i}^{\prime}(0) \lambda_{i}(0) \mathbf{r}_{i}(0)=\sum_{i \neq k} c_{i}^{\prime}(0) \lambda_{i}(0) \mathbf{r}_{i}(0)
$$

and

$$
\begin{aligned}
\left.\sum_{i=1}^{n} c_{i}(\varepsilon) \frac{d}{d \varepsilon}\left\{\lambda_{i}(\varepsilon) \mathbf{r}_{i}(\varepsilon)\right\}\right|_{\varepsilon=0} & =\left.\left\{\frac{\partial \lambda_{k}}{\partial a} a^{\prime}(0)+\nabla \lambda_{k} \cdot \mathbf{r}_{k}\right\} \mathbf{r}_{k}\right|_{U_{*}} \\
& =\left.\left\{\nabla \lambda_{k} \cdot \mathbf{r}_{k}\right\} \mathbf{r}_{k}\right|_{U_{*}} .
\end{aligned}
$$

Thus evaluating (3.4) at $\varepsilon=0$ yields

$$
f_{a} a^{\prime \prime}+\sum_{i \neq k} c_{i}^{\prime} \lambda_{i} \mathbf{r}_{i}+\left\{\nabla_{k} \cdot \mathbf{r}_{k}\right\} \mathbf{r}_{k}=0 .
$$

Multiplying both sides of (3.5) by $\mathbf{l}_{k}\left(U_{*}\right)$, we obtain that

$$
a^{\prime \prime}(0)=-\left.\frac{\nabla \lambda_{k} \cdot \mathbf{r}_{k}}{\mathbf{l}_{k} \cdot f_{a}}\right|_{U_{*}},
$$

where we have used the biorthogonality relations $\mathbf{I}_{k} \cdot \mathbf{r}_{i}=0$ for $i \neq k$ and the normalization $\mathbf{l}_{k} \cdot \mathbf{r}_{k}=1$. This completes the proof. 
The conditions in Theorem 3.1 imply that the integral curve of $\mathbf{R}_{0}$ that passes through the state $U_{*}=\left(a_{*}, u_{*}\right) \in \mathfrak{I}$ touches the hyperplane $a=a_{*}$ only at $U_{*}$ and does not cross it. Without loss of generality, we assume that $a^{\prime \prime}(0)<0$; i.e., the integral curve lies below the hyperplane $a=a_{*}$ near the state $U_{*}$ (see Fig. 1). By continuity, the above conclusions hold for all points in $\mathfrak{I}$ in a neighborhood of $U_{*}$. In particular, the integral curves of $\mathbf{R}_{0}$ passing through states $U_{0}=\left(a_{0}, u_{0}\right) \in \mathfrak{I}$ near $U_{*}$ must cross the hyperplane $a=a_{1}, a_{1}<a_{0}$ exactly twice in a neighborhood of $U_{*}$, as indicated in Fig. 1.

The solution of the Riemann problem in a neighborhood of $U_{*}$ is constructed as follows: Let $T_{t}^{i}\left(U_{L}\right)$ denote the state $t$ arclength units from $U_{L}$ along the $i$-wave curve of $U_{L}, i=1, \cdots, n$. (The $i$-wave curve of $U_{L}$ consists of all right states that can be connected to $U_{L}$ by an admissible $i$-wave [10].) Since system (1.1) is strictly hyperbolic for any $a$, all states in the image of $T^{i}\left(U_{L}\right)$ lie at level $a_{L}$. For a given value of $a_{R}$, let $T^{R}\left(U_{L}\right)$ denote the set of all right states at level $a_{R}$ that can be connected to $U_{L}$ by a solution of the Riemann problem consisting of admissible 0 -waves and $k$-waves only, and let $T_{t}^{R}\left(U_{L}\right)$ denote the point $t$ arclength units from $\mathfrak{I}$ along $T^{R}\left(U_{L}\right)$. (Choose $t$ to increase in the direction of $\lambda_{k}$ ). We say that a 0 -wave that connects $U_{L}$ to $U^{R}$ on the same integral curve of $\mathbf{R}_{0}$ by a contact discontinuity of speed zero is admissible if the integral curve of $\mathbf{R}_{0}$ does not cross the transition surface $\mathfrak{T}$ between $U_{L}$ and $U_{R}$. (Admissibility here is equivalent to conservation of the total variation of $a$ in Glimm's method (cf. [4], [9], [16]).) The curves $T^{R}\left(U_{L}\right)$ are sketched in Figs. 2 and 3. Note that $T^{R}\left(U_{L}\right)$ is a continuous curve at level $a_{R}$, but is only Lipschitz continuous due to a possible jump in the derivative at the points labeled $Q$ in Figs. 2 and 3. The continuity of the curves $T^{R}\left(U_{L}\right)$ at the special points $Q$ follows from the triple shock condition formulated in [5]. Alternatively, note that, for every $a<a_{0}$, the integral curve of $\mathbf{R}_{0}$ passing through a state $U_{0}=\left(a_{0}, u_{0}\right) \in \mathfrak{I}$ intersects the surface $a=a_{R}<a_{0}$ at exactly two points, which we can assume to be the points labeled $P$ and $Q$ in Figs. 2(a), 2(b), and 3. Thus the wave that takes $U_{L}=P$ to $U_{R}=Q$ lies in the hyperplane $a=a_{R}$ and thus must be a shock wave for the $n \times n$ system $u_{t}+f\left(a_{R}, u\right)_{x}=0$. Since

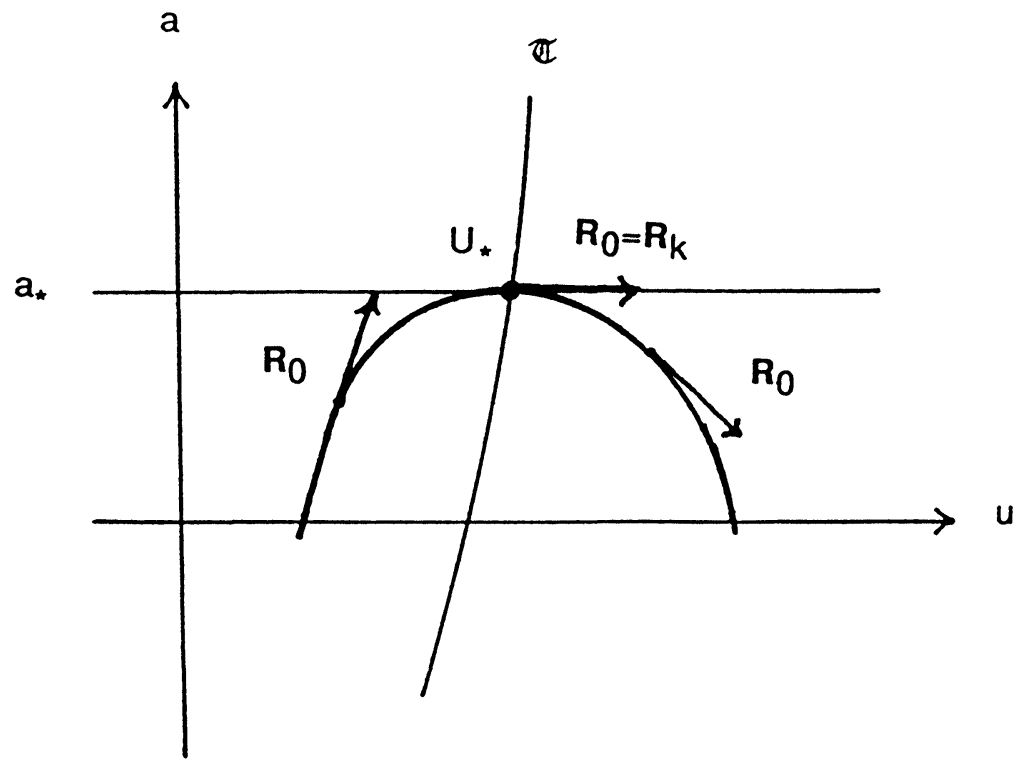

FIG. 1 


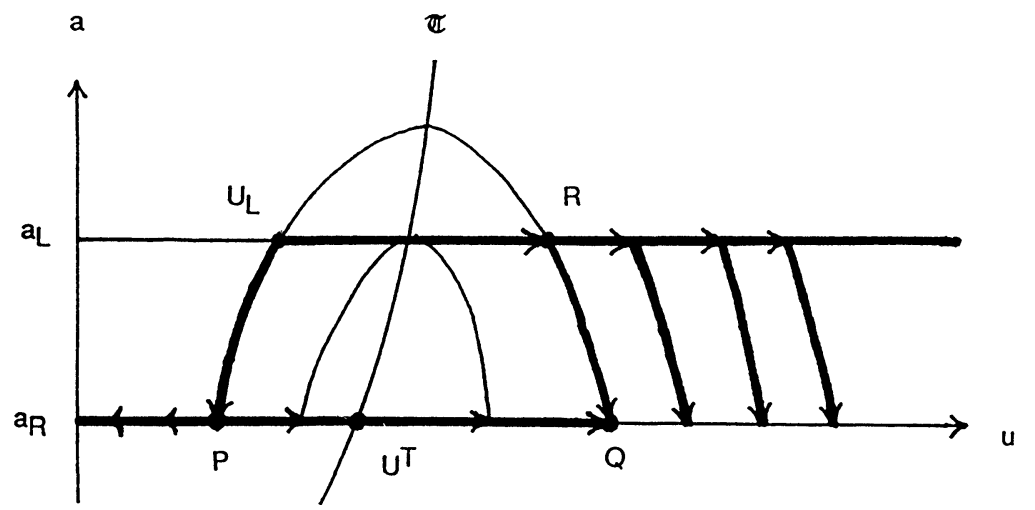

FIG. 2(a)

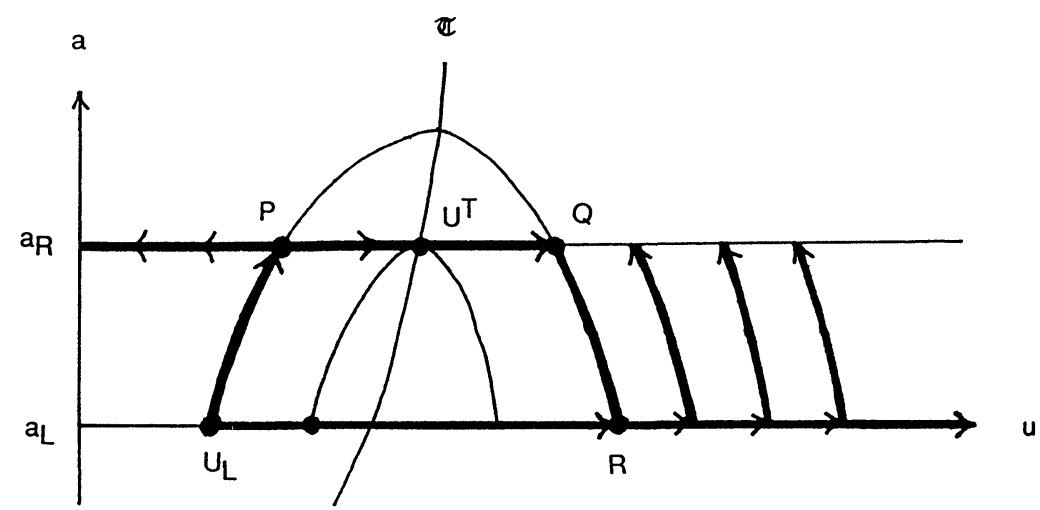

FIG. 2(b)

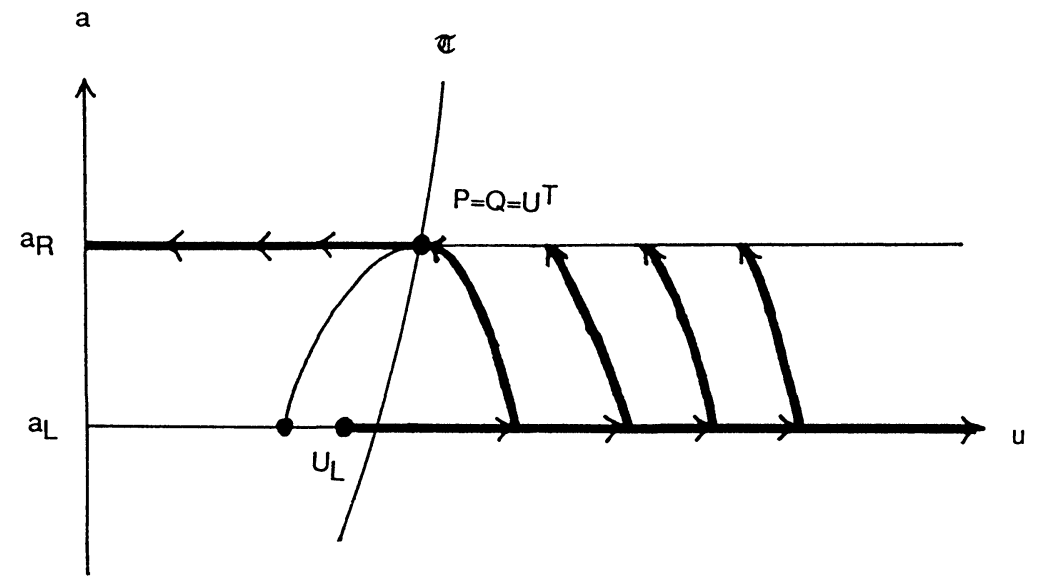

FIG. 3 
this wave is also a 0 -wave, and since $\mathbf{R}_{0}=\mathbf{R}_{k}$ at $U_{*}$, the wave from $P$ to $Q$ also must be a $k$-wave of speed zero. The continuity of $T^{R}\left(U_{L}\right)$ in $U$-space, as well as the continuous dependence of waves in $x, t$-space on $U_{R} \in T^{R}\left(U_{L}\right)$, follows directly from this observation. The existence and uniqueness of solutions of the Riemann problem for arbitrary $U_{L}$ and $U_{R}$ in a neighborhood of $U_{*}$ is accomplished by demonstrating the existence and uniqueness of values $t_{1}, \cdots, t_{n}$ such that

$$
U_{R}=T_{t_{n}}^{n} \circ \cdots \circ T_{t_{k+1}}^{k+1} \circ T_{t_{k}}^{R} \circ T_{t_{k-1}}^{k-1} \circ \cdots \circ T_{t_{1}}^{1}\left(U_{L}\right) .
$$

By definition, the elementary waves corresponding to the $T_{t}^{i}\left(U_{i}\right)$ take $U_{L}$ to $U_{R}$ as $i$ ranges from 1 to $n$, and this determines the unique solution of the Riemann problem near $U_{*}$. Since the curve $T^{R}\left(U_{L}\right)$ is only Lipschitz continuous, the implicit function theorem is difficult to apply directly to obtain existence and uniqueness of $t_{1}, \cdots, t_{n}$ for each pair $U_{L}$ and $U_{R}$ in a neighborhood of $U_{*}$. In the next two sections, we verify existence and uniqueness as a consequence of the existence and uniqueness of intersection points for Lipschitz continuous manifolds of complementary dimensions. We note that it is the Lipschitz continuity of the curves $T^{R}\left(U_{L}\right)$ that leads to the existence and uniqueness of solutions of the Riemann problem and to the continuous dependence of $x, t$-space on the left and right states $U_{L}$ and $U_{R}$. The Lipschitz continuity of the wave curves follows directly from the fact that the equations are posed in conservation form. In the true gas dynamics equations (2.1), conservation fails, and uniqueness of Riemann problem solutions, as well as continuous dependence on left and right states, is lost in a neighborhood of resonance (cf. [13]).

In conclusion, the general structure of the solutions in a neighborhood of $U_{*}$ can be described as follows: to leading order, the waves in the $0, k$-characteristic families correspond to the waves in the Riemann problem solution for the scalar inhomogeneous equation; the general solution is obtained by preceding these waves by slower waves from families $1, \cdots, k-1$ and following these waves with faster waves from families $k+1, \cdots, n$. Thus, under our generic assumptions, the Riemann problem solutions of the scalar inhomogeneous equation determine the leading-order structure of solutions in the $0, k$-family, just as the scalar homogeneous equation determines the local structure to leading order in each family of a strictly hyperbolic system.

4. Lipschitz continuous manifolds. In this section we define the notion of a $d$ dimensional Lipschitz continuous manifold in $R^{n}$ with $\varepsilon$-approximate tangent vectors. The main result of the section (Theorem 4.4) is that, under appropriate conditions, two such manifolds in $R^{n}$ with complementary dimensions intersect in a unique point. We begin by setting our notation.

For any positive integer $m$, let $R^{m}$ denote $m$-dimensional real coordinate space with the supremum norm

$$
|\mathbf{x}|=\left|\left(x_{1}, \cdots, x_{m}\right)\right| \equiv \max \left\{\left|x_{i}\right|: i=1, \cdots, m\right\} .
$$

For any $\mathbf{x}_{*} \in R^{m}$ and $\tau>0$, denote the open ball with center $\mathbf{x}_{*}$ and radius $\tau$ by

$$
B\left(\mathbf{x}_{*}, \tau\right) \equiv\left\{\mathbf{x} \in R^{m}:\left|\mathbf{x}-\mathbf{x}_{*}\right|<\tau\right\}
$$

and let

$$
I_{\tau}^{m} \equiv\left\{\mathbf{x} \in R^{m}:|\mathbf{x}|<\tau\right\}=B(\mathbf{0}, \tau) .
$$

The standard basis vectors $\mathbf{e}_{1}, \cdots, \mathbf{e}_{m} \in \boldsymbol{R}^{m}$ are given by $\mathbf{e}_{i}=(0, \cdots, 1, \cdots, 0)$, where the 1 is the $i$ th component. 
Now let $n$ and $d$ be fixed positive integers and suppose that $\mathbf{w}_{1}, \cdots, \mathbf{w}_{d}$ are linearly independent vectors in $R^{n}$. (Necessarily, $n \geqq d$ ). Since all norms on a finite-dimensional space are equivalent, there exists a constant $M_{0} \geqq 1$ that depends upon only $\mathbf{w}_{1}, \cdots, \mathbf{w}_{d}$ such that

$$
M_{0}^{-1}|\alpha| \leqq\left|\sum_{i=1}^{d} \alpha_{i} \mathbf{w}_{i}\right| \leqq M_{0}|\alpha|
$$

for all $\alpha=\left(\alpha_{1}, \cdots, \alpha_{d}\right) \in R^{d}$.

Definition 4.1. Suppose that $\varepsilon, \tau>0$, the function $\phi: I_{\tau}^{d} \rightarrow R^{n}$ is continuous, and the vectors $\mathbf{w}_{1}, \cdots, \mathbf{w}_{d}$ are linearly independent in $R^{n}$. Then $\mathbb{M}^{d} \equiv \phi\left(I_{\tau}^{d}\right)$ is called a d-dimensional Lipschitz continuous manifold with $\varepsilon$-approximate tangent vectors $\mathbf{w}_{1}, \cdots, \mathbf{w}_{d}$ if

$$
\left|\frac{\phi\left(\mathbf{t}+\mathrm{ae}_{j}\right)-\phi(\mathbf{t})}{\mathrm{a}}-\mathbf{w}_{j}\right|<\varepsilon
$$

whenever $\mathbf{t}, \mathbf{t}+\mathrm{ae}_{j} \in I_{\tau}^{d}, \mathrm{a} \neq 0$ and $1 \leqq j \leqq d$. We say also that $\phi$ defines $\mathbb{M}^{d}$.

We note that condition (4.2) is equivalent to the existence of a point $\varepsilon_{j} \in R^{n}$ that satisfies

$$
\phi\left(\mathbf{t}+\mathrm{ae}_{j}\right)=\phi(\mathbf{t})+\mathrm{aw} \mathbf{w}_{j}+\mathrm{a} \varepsilon_{j} \quad \text { with }\left|\varepsilon_{j}\right| \leqq \varepsilon .
$$

Before proving the main result, we deduce two elementary consequences of (4.2): that $\phi$ is Lipschitz continuous and that $\phi$ is $1-1$. The latter holds, provided that $\varepsilon$ is a sufficiently small positive number. First, we prove two useful estimates.

LEMMA 4.2. Assume that $\phi: I_{\tau}^{d} \rightarrow R^{n}$ satisfies condition (4.2). If $\mathbf{t}, \mathrm{t}+\alpha \in I_{\tau}^{d}$ where $\alpha=\left(\alpha_{1}, \cdots, \alpha_{d}\right)$, then

$$
|\phi(\mathbf{t}+\alpha)-\phi(\mathbf{t})| \leqq\left(M_{0}+\varepsilon d\right)|\alpha|
$$

and

$$
\left|\phi(\mathbf{t}+\alpha)-\phi(\mathbf{t})-\sum_{j=1}^{d} \alpha_{j} \mathbf{w}_{j}\right| \leqq d|\alpha| \varepsilon .
$$

Proof. The second inequality is a straightforward consequence of condition (4.2') and the identity

$$
\begin{aligned}
\phi(\mathbf{t}+\alpha)-\phi(\mathbf{t})-\sum_{j=1}^{d} \alpha_{j} \mathbf{w}_{j} & =\sum_{j=1}^{d} \phi\left(\mathbf{t}_{j}\right)-\phi\left(\mathbf{t}_{j-1}\right)-\alpha_{j} \mathbf{w}_{j} \\
& =\sum_{j=1}^{d} \phi\left(\mathbf{t}_{j-1}+\alpha_{j} \boldsymbol{e}_{j}\right)-\phi\left(\mathbf{t}_{j-1}\right)-\alpha_{j} \mathbf{w}_{j},
\end{aligned}
$$

where $\mathbf{t}_{0} \equiv \mathbf{t}$ and $\mathbf{t}_{j} \equiv \mathbf{t}+\sum_{i=1}^{j} \alpha_{i} \mathbf{e}_{i}$, since $\mathbf{t}_{j} \in I_{\tau}^{d}$ for $0 \leqq j \leqq d$. The first inequality follows from the second and the triangle inequality.

Proposition 4.3. Assume that $\phi: I_{\tau}^{d} \rightarrow R^{n}$ defines a d-dimensional Lipschitz continuous manifold $\mathbb{M}^{d}$ with $\varepsilon$-approximate tangent vectors $\mathbf{w}_{1}, \cdots, \mathbf{w}_{d}$. Then $\phi$ is Lipschitz continuous. Moreover, if

$$
\varepsilon<\left(d M_{0}\right)^{-1}
$$

then $\phi$ is $1-1$.

Proof. The Lipschitz continuity of $\phi$ follows from Lemma 4.2. To prove that $\phi$ is $1-1$, assume that there exist $\mathbf{s}, \mathbf{t} \in I_{\tau}^{d}$ such that $\phi(\mathbf{s})=\phi(\mathbf{t})$. Define $\alpha=\left(\alpha_{1}, \cdots, \alpha_{d}\right) \in$ $R^{d}$ by

$$
\mathbf{s}-\mathbf{t}=\alpha_{1} \mathbf{e}_{1}+\cdots+\alpha_{d} \mathbf{e}_{d}
$$


and set $\mathbf{t}_{0} \equiv \mathbf{t}$ and $\mathbf{t}_{j} \equiv \mathbf{t}+\sum_{i=1}^{j} \alpha_{i} \mathbf{e}_{i}$ for $1 \leqq j \leqq d$. Then $\mathbf{t}_{j} \in I_{\tau}^{d}$ for $0 \leqq j \leqq d$. Now

$$
\begin{aligned}
0=\phi(\mathbf{s})-\phi(\mathbf{t}) & =\sum_{j=1}^{d} \phi\left(\mathbf{t}_{j}\right)-\phi\left(\mathbf{t}_{j-1}\right) \\
& =\sum_{j=1}^{d} \phi\left(\mathbf{t}_{j-1}+\alpha_{j} \mathbf{e}_{j}\right)-\phi\left(\mathbf{t}_{j-1}\right) .
\end{aligned}
$$

By $\left(4.2^{\prime}\right)$, however, $\phi\left(\mathbf{t}_{j-1}+\alpha_{j} \mathbf{e}_{j}\right)-\phi\left(\mathbf{t}_{j-1}\right)=\alpha_{j} \mathbf{w}_{j}+\alpha_{j} \varepsilon_{j}$, where $\left|\varepsilon_{j}\right| \leqq \varepsilon$. Thus

$$
\sum_{j=1}^{d} \alpha_{j} \mathbf{w}_{j}=-\sum_{j=1}^{d} \alpha_{j} \varepsilon_{j}
$$

so that

$$
M_{0}^{-1}|\alpha| \leqq\left|\sum_{j=1}^{d} \alpha_{j} \mathbf{w}_{j}\right|=\left|\sum_{j=1}^{d} \alpha_{j} \varepsilon_{j}\right| \leqq \sum_{j=1}^{d}\left|\alpha_{j}\right|\left|\varepsilon_{j}\right| \leqq d|\alpha| \varepsilon .
$$

This contradicts (4.3) unless $\mathbf{s}=\mathbf{t}$.

Now consider the intersection of two affine linear subspaces (linear manifolds) $\mathbb{M}_{1}^{k}$ and $\mathbb{M}_{2}^{n-k}$ in $R^{n}$ with respective tangent vectors $\mathbf{w}_{1}, \cdots, \mathbf{w}_{k}$ and $\mathbf{w}_{k+1}, \cdots, \mathbf{w}_{n}$. Assume that $\mathbf{w}_{1}, \cdots, \mathbf{w}_{n}$ form a basis for $R^{n}$ and that $M_{0} \geqq 1$ satisfies

$$
M_{0}^{-1}|\alpha| \leqq\left|\sum_{i=1}^{n} \alpha_{i} \mathbf{w}_{i}\right| \leqq M_{0}|\alpha|
$$

Suppose that $u_{0} \in \mathbb{M}_{1}^{k}$ and $v_{0} \in \mathbb{M}_{2}^{n-k}$ and define $\alpha=\left(\alpha_{1}, \cdots, \alpha_{n}\right)$ by

$$
v_{0}-u_{0}=\sum_{i=1}^{n} \alpha_{i} \mathbf{w}_{i}
$$

Then $\mathbb{M}_{1}^{k}$ and $\mathbb{M}_{2}^{n-k}$ intersect at the unique point $u_{M} \in R^{n}$ defined by

$$
u_{M}=u_{0}+\sum_{i=1}^{k} \alpha_{i} \mathbf{w}_{i}=v_{0}-\sum_{i=k+1}^{n} \alpha_{i} \mathbf{w}_{i}
$$

Moreover, the distance between the intersection point and the known points on the manifolds can be estimated by

$$
\left|u_{M}-u_{0}\right|=\left|\sum_{i=1}^{k} \alpha_{i} \mathbf{w}_{i}\right| \leqq M_{0}|\alpha| \leqq M_{0}^{2}\left|v_{0}-u_{0}\right|,
$$

with a similar estimate for $\left|u_{M}-v_{0}\right|$.

In the case of nonlinear manifolds, the point $u_{M}$, defined above, gives only an approximation to the point of intersection, and it may not lie in either of the manifolds. Consequently, we find the intersection by "projecting" $u_{M}$ onto the manifolds and then iterating this construction.

THEOREM 4.4. Let

$$
\phi: I_{\tau}^{k} \rightarrow R^{n}, \quad \psi: I_{\tau}^{n-k} \rightarrow R^{n}
$$

define Lipschitz continuous manifolds $\mathbb{M}_{1}^{k}$ and $\mathbb{M}_{2}^{n-k}$ with $\varepsilon$-approximate tangent vectors $\mathbf{w}_{1}, \cdots, \mathbf{w}_{k}$ and $\mathbf{w}_{k+1}, \cdots, \mathbf{w}_{n}$, respectively, where $\mathbf{w}_{1}, \cdots, \mathbf{w}_{n}$ form a basis for $R^{n}$. Assume that

$$
\varepsilon<\frac{1}{2 n M_{0}}
$$


and that there is a state $u_{*} \in R^{n}$, a number $\rho$ with $0<\rho<1$, and points $\mathbf{p}_{0} \in I_{\rho \tau}^{k}$ and $\mathbf{q}_{0} \in I_{\rho \tau}^{n-k}$ satisfying

$$
\phi\left(\mathbf{p}_{0}\right), \psi\left(\mathbf{q}_{0}\right) \in B\left(u_{*}, \delta\right)
$$

for some positive number $\delta \leqq(1-\rho) \tau /\left(4 M_{0}\right)$. Then $\mathbb{M}_{1}^{k}$ and $\mathbb{M}_{2}^{n-k}$ intersect at a unique point, and the intersection point lies in the ball $B\left(u_{*}, \gamma\right)$, where

$$
\gamma=\gamma(\delta) \equiv 6 M_{0}^{2} \delta \text {. }
$$

Proof. First, note that $I_{\tau}^{k} \times I_{\tau}^{n-k}=I_{\tau}^{n}$. Now define $\alpha: I_{\tau}^{n} \rightarrow R^{n}$ as follows: for any point $(\mathbf{p} ; \mathbf{q}) \in I_{\tau}^{k} \times I_{\tau}^{n-k}$, the point $\alpha=\alpha(\mathbf{p} ; \mathbf{q}) \equiv\left(\alpha_{1}, \cdots, \alpha_{n}\right)$ is given by

$$
\psi(\mathbf{q})-\phi(\mathbf{p})=\sum_{i=1}^{n} \alpha_{i} \mathbf{w}_{i}
$$

Also, define $\Phi: I_{\tau}^{n} \rightarrow R^{n}$ by

$$
\Phi(\mathbf{p} ; \mathbf{q}) \equiv\left(\mathbf{p}+\alpha^{1} ; \mathbf{q}-\alpha^{2}\right)
$$

where $\quad \alpha(\mathbf{p} ; \mathbf{q})=\left(\alpha^{1}(\mathbf{p} ; \mathbf{q}) ; \alpha^{2}(\mathbf{p} ; \mathbf{q})\right)=\left(\alpha_{1}, \cdots, \alpha_{k} ; \alpha_{k+1}, \cdots, \alpha_{n}\right)$ and let $u_{M}=$ $u_{M}(\mathbf{p} ; \mathbf{q}) \in R^{n}$ denote the approximate intersection point of the manifolds

$$
u_{M} \equiv \phi(\mathbf{p})+\sum_{i=1}^{k} \alpha_{i} \mathbf{w}_{i}=\psi(\mathbf{q})-\sum_{i=k+1}^{n} \alpha_{i} \mathbf{w}_{i}
$$

It follows that the manifolds $\mathbb{M}_{1}^{k}$ and $\mathbb{M}_{2}^{n-k}$ intersect at $\phi(\mathbf{p})=\psi(\mathbf{q})$ if and only if $\alpha(\mathbf{p} ; \mathbf{q})=\mathbf{0}$ if and only if $(\mathbf{p} ; \mathbf{q})$ is a fixed point of $\Phi$. We will show that $\Phi$ has a unique fixed point.

First, we prove the existence of a fixed point by iterating $\Phi$. For each $(\mathbf{p} ; \mathbf{q}) \in$ $I_{\tau}^{k} \times I_{\tau}^{n-k},(4.4)$ yields

$$
|\alpha(\mathbf{p} ; \mathbf{q})| \leqq M_{0}\left|\sum_{i=1}^{n} \alpha_{i} \mathbf{w}_{i}\right|=M_{0}|\psi(\mathbf{q})-\phi(\mathbf{p})|,
$$

so that

$$
\begin{aligned}
|\Phi(\mathbf{p} ; \mathbf{q})-(\mathbf{p} ; \mathbf{q})| & =\left|\left(\alpha^{1} ;-\alpha^{2}\right)\right| \\
& =|\alpha(\mathbf{p} ; \mathbf{q})| \leqq M_{0}|\psi(\mathbf{q})-\phi(\mathbf{p})| .
\end{aligned}
$$

If $\Phi(\mathbf{p} ; \mathbf{q}) \in I_{\tau}^{n}$, then Lemma 4.2 implies that

$$
\begin{aligned}
& \phi\left(\mathbf{p}+\alpha^{1}\right)=\phi(\mathbf{p})+\sum_{i=1}^{k} \alpha_{i} \mathbf{w}_{i}+\varepsilon_{1} \quad \text { with }\left|\varepsilon_{1}\right| \leqq k\left|\alpha^{1}\right| \varepsilon \\
& \psi\left(\mathbf{q}-\alpha^{2}\right)=\psi(\mathbf{q})-\sum_{i=k+1}^{n} \alpha_{i} \mathbf{w}_{i}+\varepsilon_{2} \quad \text { with }\left|\varepsilon_{2}\right| \leqq(n-k)\left|\alpha^{2}\right| \varepsilon
\end{aligned}
$$

Therefore $\phi\left(\mathbf{p}+\alpha^{1}\right)=u_{M}(\mathbf{p} ; \mathbf{q})+\varepsilon_{1}$ and $\psi\left(\mathbf{q}-\alpha^{2}\right)=u_{M}(\mathbf{p} ; \mathbf{q})+\varepsilon_{2}$. Consequently,

$$
\begin{aligned}
\left|\psi\left(\mathbf{q}-\alpha^{2}\right)-\phi\left(\mathbf{p}+\alpha^{1}\right)\right| & \leqq\left|\varepsilon_{1}\right|+\left|\varepsilon_{2}\right| \leqq k\left|\alpha^{1}\right| \varepsilon+(n-k)\left|\alpha^{2}\right| \varepsilon \\
& \leqq n \varepsilon|\alpha| \leqq\left(n M_{0} \varepsilon\right)|\psi(\mathbf{q})-\phi(\mathbf{p})| .
\end{aligned}
$$

Now define the sequence $\left\{\left(\mathbf{p}_{m} ; \mathbf{q}_{m}\right)\right\}$ for $m=0,1,2, \cdots$ by

$$
\left(\mathbf{p}_{m+1} ; \mathbf{q}_{m+1}\right) \equiv \Phi\left(\mathbf{p}_{m} ; \mathbf{q}_{m}\right) \text {. }
$$


(For now, we assume that the sequence is well defined; that is, $\left(\mathbf{p}_{m} ; \mathbf{q}_{m}\right) \in I_{\tau}^{k} \times I_{\tau}^{n-k}$ for all $m$. We verify this later.) We show that the sequence is a Cauchy sequence. From (4.9) and (4.10) we have that

$$
\begin{aligned}
\left|\left(\mathbf{p}_{m+1} ; \mathbf{q}_{m+1}\right)-\left(\mathbf{p}_{m} ; \mathbf{q}_{m}\right)\right| & =\left|\Phi\left(\mathbf{p}_{m} ; \mathbf{q}_{m}\right)-\left(\mathbf{p}_{m} ; \mathbf{q}_{m}\right)\right| \\
& \leqq M_{0}\left|\psi\left(\mathbf{q}_{m}\right)-\phi\left(\mathbf{p}_{m}\right)\right|
\end{aligned}
$$

and

$$
\left|\psi\left(\mathbf{q}_{m+1}\right)-\phi\left(\mathbf{p}_{m+1}\right)\right| \leqq\left(n M_{0} \varepsilon\right)\left|\psi\left(\mathbf{q}_{m}\right)-\phi\left(\mathbf{p}_{m}\right)\right| .
$$

The second inequality can be iterated to yield

$$
\left|\psi\left(\mathbf{q}_{m}\right)-\phi\left(\mathbf{p}_{m}\right)\right| \leqq\left(n M_{0} \varepsilon\right)^{m}\left|\psi\left(\mathbf{q}_{0}\right)-\phi\left(\mathbf{p}_{0}\right)\right|,
$$

and then the first inequality implies that, for any $m \geqq 0, s \geqq 1$,

$$
\begin{aligned}
\left|\left(\mathbf{p}_{m+s} ; \mathbf{q}_{m+s}\right)-\left(\mathbf{p}_{m} ; \mathbf{q}_{m}\right)\right| & \leqq \sum_{j=m}^{m+s-1}\left|\left(\mathbf{p}_{j+1} ; \mathbf{q}_{j+1}\right)-\left(\mathbf{p}_{j} ; \mathbf{q}_{j}\right)\right| \\
& \leqq \sum_{j=m}^{m+s-1} M_{0}\left|\psi\left(\mathbf{q}_{j}\right)-\phi\left(\mathbf{p}_{j}\right)\right| \\
& \leqq\left(\sum_{j=m}^{m+s-1}\left(n M_{0} \varepsilon\right)^{j}\right) M_{0}\left|\psi\left(\mathbf{q}_{0}\right)-\phi\left(\mathbf{p}_{0}\right)\right| \\
& \leqq \frac{\left(n M_{0} \varepsilon\right)^{m}}{1-n M_{0} \varepsilon} M_{0}\left|\psi\left(\mathbf{q}_{0}\right)-\phi\left(\mathbf{p}_{0}\right)\right|,
\end{aligned}
$$

provided that $n M_{0} \varepsilon<1$. This proves that the sequence is a Cauchy sequence. Denote the limit by $\left(\mathbf{p}_{\infty} ; \mathbf{q}_{\infty}\right)$ and assume that $\left(\mathbf{p}_{\infty} ; \mathbf{q}_{\infty}\right) \in I_{\tau}^{k} \times I_{\tau}^{n-k}$ (we verify this below). Since $\phi$ and $\psi$ are continuous, $(4.10)_{m}$ implies that $\psi\left(\mathbf{q}_{\infty}\right)=\phi\left(\mathbf{p}_{\infty}\right)$, so that $\left(\mathbf{p}_{\infty} ; \mathbf{q}_{\infty}\right)$ is a fixed point of $\Phi$.

To complete the proof of existence, we must show that the sequence is well defined and that its limit lies in the domain of $\Phi$. Assume that $\left(\mathbf{p}_{0} ; \mathbf{q}_{0}\right), \cdots,\left(\mathbf{p}_{m-1} ; \mathbf{q}_{m-1}\right)$ are in $I_{\tau}^{k} \times I_{\tau}^{n-k}$. Then $\left(\mathbf{p}_{m} ; \mathbf{q}_{m}\right)$ is well defined, and, from (4.11), we obtain that

$$
\left|\left(\mathbf{p}_{m} ; \mathbf{q}_{m}\right)-\left(\mathbf{p}_{0} ; \mathbf{q}_{0}\right)\right| \leqq \frac{1}{1-n M_{0} \varepsilon} M_{0}\left|\psi\left(\mathbf{q}_{0}\right)-\phi\left(\mathbf{p}_{0}\right)\right|
$$

Consequently,

$$
\left|\left(\mathbf{p}_{m} ; \mathbf{q}_{m}\right)\right| \leqq\left|\left(\mathbf{p}_{0} ; \mathbf{q}_{0}\right)\right|+\frac{1}{1-n M_{0} \varepsilon} M_{0}\left|\psi\left(\mathbf{q}_{0}\right)-\phi\left(\mathbf{p}_{0}\right)\right|
$$

and hence

$$
\left|\left(\mathbf{p}_{\infty} ; \mathbf{q}_{\infty}\right)\right| \leqq\left|\left(\mathbf{p}_{0} ; \mathbf{q}_{0}\right)\right|+\frac{1}{1-n M_{0} \varepsilon} M_{0}\left|\psi\left(\mathbf{q}_{0}\right)-\phi\left(\mathbf{p}_{0}\right)\right| .
$$

The result follows from the choice of $\varepsilon$ and $\delta$, since $\left|\psi\left(\mathbf{q}_{0}\right)-\phi\left(\mathbf{p}_{0}\right)\right|<2 \delta$.

The estimate on the location of the intersection point is obtained as follows. From (4.11) we obtain that

$$
\left|\left(\mathbf{p}_{\infty} ; \mathbf{q}_{\infty}\right)-\left(\mathbf{p}_{0} ; \mathbf{q}_{0}\right)\right| \leqq \frac{1}{1-n M_{0} \varepsilon} M_{0}\left|\psi\left(\mathbf{q}_{0}\right)-\psi\left(\mathbf{p}_{0}\right)\right|<4 \mathbf{M}_{0} \delta
$$


Therefore, by Lemma 4.2,

$$
\left|\phi\left(\mathbf{p}_{\infty}\right)-\phi\left(\mathbf{p}_{0}\right)\right| \leqq\left(M_{0}+\varepsilon k\right) 4 M_{0} \delta,
$$

so that

$$
\left|\phi\left(\mathbf{p}_{\infty}\right)-u_{*}\right| \leqq\left(M_{0}+\varepsilon k\right) 4 M_{0} \delta+\delta \leqq 6 M_{0}^{2} \delta .
$$

Now we show that $\Phi$ has at most one fixed point. Assume that there are two fixed points $\left(\mathbf{p}_{1} ; \mathbf{q}_{1}\right)$ and $\left(\mathbf{p}_{2} ; \mathbf{q}_{2}\right)$. Then

$$
\phi\left(\mathbf{p}_{1}\right)=\psi\left(\mathbf{q}_{1}\right) \text { and } \phi\left(\mathbf{p}_{2}\right)=\psi\left(\mathbf{q}_{2}\right)
$$

However,

$$
\begin{gathered}
\phi\left(\mathbf{p}_{2}\right)-\phi\left(\mathbf{p}_{1}\right)=\sum_{i=1}^{k} \alpha_{i} \mathbf{w}_{i}+\varepsilon_{1}, \\
\psi\left(\mathbf{q}_{2}\right)-\psi\left(\mathbf{q}_{1}\right)=\sum_{i=k+1}^{n} \alpha_{i} \mathbf{w}_{i}+\varepsilon_{2},
\end{gathered}
$$

where $\alpha=\left(\mathbf{p}_{2}-\mathbf{p}_{1} ; \mathbf{q}_{2}-\mathbf{q}_{1}\right)$ and $\left|\varepsilon_{1}\right| \leqq k|\alpha| \varepsilon,\left|\varepsilon_{2}\right| \leqq(n-k)|\alpha| \varepsilon$. Subtracting yields

$$
M_{0}^{-1}|\alpha| \leqq\left|\sum_{i=1}^{k} \alpha_{i} \mathbf{w}_{i}-\sum_{i=k+1}^{n} \alpha_{i} \mathbf{w}_{i}\right| \leqq n|\alpha| \varepsilon,
$$

and this contradicts (4.6) unless $\alpha=\mathbf{0}$. This completes the proof of uniqueness.

5. The Riemann problem in a neighborhood of $\mathfrak{T}$. Choose $U_{*}=\left(a_{*}, u_{*}\right) \in \mathfrak{I}$. Since $a$ is constant along $i$-wave curves, $i=1, \cdots, n$, it is convenient for us to study the solution in $u$-space $R^{n}$ instead of $U=(a, u)$-space $R^{n+1}$. Thus let $\mathbf{r}_{1}, \cdots, \mathbf{r}_{n}$ denote the eigenvectors of $\mathrm{df}$ at $U_{*}$. Let $\mathbf{v}_{1}, \cdots, \mathbf{v}_{n-1}$ denote an orthonormal basis for the tangent space of $\mathfrak{I}\left(a_{*}\right)$, where $\mathfrak{T}(a)$ is the $(n-1)$-dimensional surface in $R^{n}$ defined by $\mathfrak{I}(a) \equiv\left\{u \in R^{n}:(a, u) \in \mathfrak{I}\right\}$. Thus

$$
\mathbf{v}_{1}, \cdots, \mathbf{v}_{n-1}, \mathbf{r}_{k}
$$

is a basis for $R^{n}$. Let $P u$ denote the projection of $u$ onto $\operatorname{span}\left\{\mathbf{v}_{1}, \cdots, \mathbf{v}_{n-1}\right\}$ along $\mathbf{r}_{k}$. For $U_{L}=\left(a_{L}, u_{L}\right)$, let $\Gamma_{i}\left(U_{L}\right)$, a subset of $R^{n}$, denote the $i$-wave curve of $u_{L}$ at level $a_{L}$. For $a_{L}$ fixed, we let $T^{i}\left(u_{L}, t\right) \equiv T_{t}^{i}\left(u_{L}\right)$ denote the point in $R^{n}$ that is $t$ arclength units from $u_{L}$ along $\Gamma_{i}\left(U_{L}\right)$, where we take $t$ to be positive along the rarefaction curve and negative along the shock curve. For fixed $a_{L}$ and $a_{R}$, let $\Gamma_{k}^{R}\left(U_{L}\right)$ in $R^{n}$ denote the $k$-wave curve of $U_{L}$ at level $a_{R}$; i.e., the set of all states $u_{R}$ such that the Riemann problem $\left[U_{L}, U_{R}\right]$ is solvable by a 0 -wave and a $k$-wave only. $\left(\Gamma_{k}^{R}\left(U_{L}\right)\right.$ is a subset of $R^{n}$ that is determined by the choice of $\left.a_{R}\right)$. For fixed $a_{L}$ and $a_{R}$, we let

$$
T^{R}\left(u_{L}, t\right) \equiv T_{t}^{R}\left(u_{L}\right)
$$

denote the state $u_{R} \in \Gamma_{k}^{R}\left(U_{L}\right)$, which is $t$ arclength units from the state $u^{T} \equiv u^{T}\left(u_{L}\right)$, where $u^{T}\left(u_{L}\right)$ is given by

$$
u^{T}\left(u_{L}\right)=\Gamma_{k}^{R}\left(U_{L}\right) \cap \mathfrak{I}\left(a_{R}\right) .
$$

In other words, $u^{T}$ is the point in $R^{n}$ at which the curve $T^{R}\left(U_{L}, \cdot\right)$ intersects the transition surface at level $a_{R}$. Again, the function $u^{T}\left(u_{L}\right)$ is determined by a fixed choice of $a_{L}$ and $a_{R}$.

For fixed $a_{L}$ and $a_{R}$, now consider the functions $T^{i}\left(u_{L}, t\right), u^{T}\left(u_{L}\right)$, and $T^{R}\left(u_{L}, t\right)$. Since (1.1) is strictly hyperbolic and genuinely nonlinear at fixed $a$, we know that $T^{i}$ is a $C^{2}$ function of its arguments. As we noted in $\S 2, u^{T}$ is defined in terms of smoothly 
varying integral curves and shock curves, and so it also is a $C^{2}$ function of its argument $u_{L}$. Moreover, the function $T^{R}\left(u_{L}, t\right)$ is continuously differentiable except at values of $t$ corresponding to the point $Q$ in Figs. 2 and 3. At this point, $T^{R}\left(u_{L}, \cdot\right)$ is only Lipschitz continuous with a jump in the derivative bounded by $C_{1}\left|a_{L}-a_{R}\right|$. Now let $u_{L}, u_{R}, a_{L}$, and $a_{R}$ be fixed. For $\mathbf{t}=(\mathbf{p} ; \mathbf{q})=\left(t_{1}, \cdots, t_{k} ; t_{k+1}, \cdots, t_{n}\right)$, define the functions $\phi(\mathbf{p})$ and $\psi(\mathbf{q})$ as follows:

$$
\begin{aligned}
& \phi(\mathbf{p})=T_{t_{k}}^{R} \circ \ldots \circ T_{t_{1}}^{1}\left(u_{L}\right), \\
& \psi(\mathbf{q})=T_{t_{k+1}}^{-(k+1)} \circ \ldots \circ T_{t_{n}}^{-n}\left(u_{R}\right),
\end{aligned}
$$

where $T_{t}^{-j}$ denotes the inverse of $T_{t}^{j}$. Our aim is to show that in some neighborhood of $u_{*}, \phi$ and $\psi$ define Lipschitz continuous manifolds in $R^{n}$ with $\varepsilon$-approximate tangent vectors $\mathbf{w}_{1}, \cdots, \mathbf{w}_{n}$, where

$$
\mathbf{w}_{j}= \begin{cases}P \mathbf{r}_{j} & \text { for } j<k ; \\ \mathbf{r}_{j} & \text { for } j \geqq k .\end{cases}
$$

We can then apply Theorem 4.4 by showing that $\varepsilon<1 / 2 n M_{0}$ when we restrict to states close enough to $U_{*}$.

LEMMA 5.1. There exist positive constants $\sigma_{1}, \delta_{1}$, and $C_{2}$ such that, if $a_{L}$ and $a_{R}$ lie in $\left\{a:\left|a-a_{*}\right|<\sigma<\sigma_{1}\right\}$ and $u$ and $v$ are arbitrary states in $B\left(u_{*}, \delta\right), 0<\delta<\delta_{1}$, then

$$
u^{T}(u)-u^{T}(v)=P(u-v)+\varepsilon_{2}
$$

where

$$
\left|\varepsilon_{2}\right| \leqq C_{2}(\sigma+\delta)|u-v|
$$

Proof. This follows directly from the fact that $u^{T}$ is smooth and that $u^{T}$ lies on the smooth surface $\mathfrak{T}$. (By definition, $u^{T}$ is obtained approximately by projecting onto $\mathfrak{I}$ along $\mathbf{R}_{0}$.)

LEMMA 5.2. There exist positive constants $\tau_{2}, \sigma_{2}<\sigma_{1}, \delta_{2}<\delta_{1}$, and $C_{1}$ such that, if $0<\delta<\delta_{2}$ and $a_{L}$ and $a_{R}$ lie in $\left\{a:\left|a-a_{*}\right|<\sigma<\sigma_{2}\right\}$, then $u^{T}$ is defined for all $u$ in $B\left(u_{*}, \delta\right), T^{R}$ is defined for all $(u, t)$ in $B\left(u_{*}, \delta\right) \times I_{\tau}^{1}$ with

$$
T^{R}(u, t)-T^{R}(v, t)=u^{T}(u)-u^{T}(v)+\varepsilon,
$$

where

$$
|\varepsilon| \leqq C_{1}(\sigma+\delta)|t| .
$$

Proof. This follows directly from the fact that the tangent vector to $T^{R}\left(u_{L}, \cdot\right)$ is equal to $\mathbf{r}_{k}$ to within an error of $O(1)(\sigma+\delta)$ (see Figs. 1-3).

Now set $u_{m}(\mathrm{t}) \equiv T_{t_{n}}^{n} \circ \cdots \circ T_{t_{1}}^{1}\left(u_{L}\right)$. Then, for each positive constant $\delta<\delta_{2}$, there exist positive constants $\delta^{\prime}<\delta, \sigma<\sigma_{2}$, and $\tau<\tau_{2}$ such that if $a_{L} \in\left\{a:\left|a-a_{*}\right|<\sigma\right\}$ and $u_{L} \in B\left(u_{*}, \delta^{\prime}\right)$, then the Riemann problem at level $a_{L}$ is defined, takes values in $B\left(u_{*}, \delta\right)$, and satisfies the condition

$$
\frac{u_{m}\left(\mathbf{t}+\alpha \mathbf{e}_{j}\right)-u_{m}(\mathbf{t})}{\alpha}=\mathbf{R}_{j}+O(1)(\sigma+\delta)
$$

whenever $\mathbf{t}, \mathbf{t}+\alpha \mathbf{e}_{j} \in I_{\tau}^{n}$ for $\alpha \neq 0$ and $j=1, \cdots, n$. The existence of constants $\delta, \sigma$, and $\tau$ follows directly from the local properties of the Riemann problem in strictly hyperbolic systems because (1.3) defines a strictly hyperbolic system at each level $a_{L}$, and these systems depend smoothly on $a_{L}$. 
LeMmA 5.3. Let $\delta^{\prime}<\delta<\delta_{2}, \sigma$ and $\tau$ be chosen so that (5.2) holds. Suppose that $u_{L}, u_{R} \in B\left(u_{*}, \delta\right)$ and assume that $a_{L}, a_{R} \in\left\{a:\left|a-a_{*}\right|<\sigma\right\}$. Then there exists a positive constant $C_{3}$ such that

$$
\left|\frac{\phi\left(\mathbf{p}+\alpha \mathbf{e}_{j}\right)-\phi(\mathbf{p})}{\alpha}-\mathbf{w}_{j}\right| \leqq C_{3}(\sigma+\delta)
$$

whenever $\mathbf{p}, \mathbf{p}+\alpha \mathbf{e}_{j} \in I_{\tau}^{k}$ for $\alpha \neq 0$ and $1 \leqq j \leqq k$.

Proof. First, for $j=k$, let

$$
u_{M}=T_{t_{k-1}}^{k-1} \circ \cdots \circ T_{t_{1}}^{1}\left(u_{L}\right)
$$

Then

$$
\phi\left(\mathbf{p}+\alpha \mathbf{e}_{k}\right)=T_{t_{k}+\alpha}^{R}\left(u_{M}\right)=T^{R}\left(u_{M}, t_{k}+\alpha\right)
$$

and

$$
\phi(\mathbf{p})=T_{t_{k}}^{R}\left(u_{M}\right)=T^{R}\left(u_{M}, t_{k}\right) .
$$

Thus (5.3) follows directly from the Lipschitz continuity of $T^{R}\left(u_{L}, \cdot\right)$ in this case. Now consider the case where $j<k$. Then, letting $\mathbf{p}^{\prime}=\left(t_{1}, \cdots, t_{k-1}\right)$ and

$$
u_{m}\left(\mathbf{p}^{\prime}\right)=T_{t_{k-1}}^{k-1} \circ \cdots \circ T_{t_{1}}^{1}\left(u_{L}\right)
$$

it follows from (5.2) that

$$
\frac{u_{m}\left(\mathbf{p}^{\prime}+\alpha \mathbf{e}_{j}\right)-u_{m}\left(\mathbf{p}^{\prime}\right)}{\alpha}=\mathbf{r}_{j}+O(1) \delta
$$

By Lemma 5.1,

$$
\begin{aligned}
\frac{u^{T} \circ u_{m}\left(\mathbf{p}^{\prime}+\alpha \mathbf{e}_{j}\right)-u^{T} \circ u_{m}\left(\mathbf{p}^{\prime}\right)}{\alpha} & =P\left(\frac{u_{m}\left(\mathbf{p}^{\prime}+\alpha \mathbf{e}_{j}\right)-u_{m}\left(\mathbf{p}^{\prime}\right)}{\alpha}\right)+O(1)(\sigma+\delta) \\
& =P \mathbf{r}_{j}+O(1)(\sigma+\delta) \\
& =\mathbf{w}_{j}+O(1)(\sigma+\delta) .
\end{aligned}
$$

Thus, by Lemma 5.2,

$$
\begin{aligned}
\frac{\phi\left(\mathbf{p}+\alpha \mathbf{e}_{j}\right)-\phi(\mathbf{p})}{\alpha} & =\frac{T^{R}\left(u_{m}\left(\mathbf{p}^{\prime}+\alpha \mathbf{e}_{j}\right), t_{k}\right)-T^{R}\left(u_{m}\left(\mathbf{p}^{\prime}\right), t_{k}\right)}{\alpha} \\
& =P\left(\frac{u_{m}\left(\mathbf{p}^{\prime}+\alpha \mathbf{e}_{j}\right)-u_{m}\left(\mathbf{p}^{\prime}\right)}{\alpha}\right)+O(1)(\sigma+\delta) \\
& =\mathbf{w}_{j}+O(1)(\sigma+\delta)
\end{aligned}
$$

as desired.

We have the following theorem.

THEOREM 5.4. For each positive constant $\delta<\delta_{2}$, there exist positive constants $C$, $\delta^{\prime}<\delta, \sigma$, and $\tau$ such that, if $u_{L}$ and $u_{R}$ are states in $B\left(u_{*}, \delta^{\prime}\right)$ and if $a_{L}$ and $a_{R}$ lie in $\left\{a:\left|a-a_{*}\right|<\sigma\right\}$, then the mappings $\phi$ and $\psi$ given in (5.1) for $|\mathbf{t}|<\tau$ define Lipschitz continuous manifolds $\mathbb{M}_{1}^{k}$ and $\mathbb{M}_{2}^{n-k}$ with $\varepsilon$-approximate tangent vectors $\mathbf{w}_{1}, \cdots, \mathbf{w}_{k}$ and $\mathbf{w}_{k+1}, \cdots, \mathbf{w}_{n}$, respectively, where $\varepsilon \leqq C(\sigma+\delta)$. Moreover, $\phi$ and $\psi$ take values in $B\left(u_{*}, \delta\right)$.

Proof. Since the wave curves for a strictly hyperbolic system depend smoothly on left and right states, $\psi$ defines a $C^{2}$ manifold for each $u_{R}$ in a neighborhood of $u_{*}$, and thus, in the case of $\psi$, the result follows directly from (5.2). The result for $\phi$ follows directly from Lemma 5.3. 
Now choose $\delta_{3}<\delta_{3}^{\prime}$ and $\sigma_{3}$ small enough so that $\varepsilon=C\left(\sigma_{3}+\delta_{3}\right)<1 / 2 n M_{0}$, where, again, $M_{0}$ is chosen to satisfy the condition

$$
M_{0}^{-1}|\alpha| \leqq\left|\sum_{i=1}^{n} \alpha_{i} \mathbf{w}_{i}\right| \leqq M_{0}|\alpha| .
$$

Then, for $|\mathbf{t}|<\tau_{3}, \phi$ and $\psi$ define Lipschitz continuous manifolds $M_{1}^{k}$ and $M_{2}^{n-k}$ with $\varepsilon$-approximate tangent vectors $\mathbf{w}_{1}, \cdots, \mathbf{w}_{k}$ and $\boldsymbol{w}_{k+1}, \cdots, \mathbf{w}_{n}$, respectively; so, by Theorem 4.4, there exists a positive constant $\delta$ (which must be chosen small depending upon $\tau_{3}$ ) such that, if

$$
\mathbb{M}_{1}^{k} \cap B\left(u_{*}, \delta\right) \neq \varnothing \quad \text { and } \quad \mathbb{M}_{2}^{n-k} \cap B\left(u_{*}, \delta\right) \neq \varnothing,
$$

then $\mathbb{M}_{1}^{k}$ and $\mathbb{M}_{2}^{n-k}$ intersect at a unique point in $B\left(u_{*}, \gamma\right)$, where

$$
\gamma=\gamma(\delta) \equiv 8 M_{0}^{2} \delta .
$$

We can now prove the following theorem, which gives the existence and uniqueness of solutions of the Riemann problem in a neighborhood of the state $U_{*}$.

THEOREM 5.5. If $u_{L}$ and $u_{R}$ each lie in $B\left(u_{*}, \delta\right)$, and if $\left|a_{L}-a_{*}\right|<\sigma_{3},\left|a_{R}-a_{*}\right|<\sigma_{3}$, then there exists a unique solution of the Riemann problem in the class of admissible 0 -waves, shock waves, and rarefaction waves taking $u$-values in $B\left(u_{*}, \delta_{3}\right)$.

Proof. We have that $\mathbb{M}_{1}^{k} \cap \mathbb{M}_{2}^{n-k}=\left\{u_{M}\right\}$, where $u_{M}$ lies in $B\left(u_{*}, \delta_{3}\right)$. Thus, by (5.1),

$$
u_{M}=T_{t_{k}}^{R} \circ \cdots \circ T_{t_{1}}^{1}\left(u_{L}\right)
$$

and

$$
u_{M}=T_{t_{k+1}}^{-(k+1)} \circ \cdots \circ T_{t_{n}}^{-n}\left(u_{R}\right),
$$

for some $\mathbf{t} \in R^{n}$, where $|\mathbf{t}|<\tau_{3}$ by our choice of $\delta \ll 1$. Thus, setting $U_{L}=\left(a_{L}, u_{L}\right)$, $U_{M}=\left(a_{M}, u_{M}\right)$, and $U_{R}=\left(a_{R}, u_{R}\right),(5.5)$ defines the unique solution of the Riemann problem $\left[U_{L}, U_{M}\right]$ in the class of 0 -through $k$-waves taking $u$-values in $B\left(u_{*}, \delta_{3}\right)$; and (5.6) defines the unique solution of the Riemann problem $\left[U_{M}, U_{R}\right]$ in the class of $(k+1)$-through $n$-waves taking $u$-values in $B\left(u_{*}, \delta_{3}\right)$. The concatenation of these solutions gives the unique solution of the Riemann problem $\left[U_{L}, U_{R}\right]$ in $B\left(u_{*}, \delta_{3}\right)$, since then

$$
u_{R}=T_{t_{n}}^{n} \circ \cdots \circ T_{t_{k}}^{R} \circ \cdots \circ T_{t_{1}}^{1} u_{L} .
$$

This completes the proof of Theorem 3.1.

Acknowledgments. We thank Dan Marchesin and Jorge Patino for showing us the Lagrangian transformation and for open discussions on these systems. In addition, we thank Professors Wang Jinghua and Lin Longwei for reading the original manuscript and making helpful comments.

\section{REFERENCES}

[1] R. Courant ANd K. O. Friedrichs, Supersonic Flow and Shock Waves, John Wiley, New York, 1948.

[2] J. GlimM, Solutions in the large for nonlinear hyperbolic systems of equations, Comm. Pure Appl. Math., 18 (1965), pp. 697-715.

[3] S. K. GoDUNOV, A difference method for numerical calculations of discontinuous solutions of the equations of hydrodynamics, Mat. Sb., 47 (1959), pp. 271-306. (In Russian).

[4] E. ISAACSON, Global solution of a Riemann problem for a non-strictly hyperbolic system of conservation laws arising in enhanced oil recovery, preprint, Rockefeller University, New York, 1981. 
[5] E. IsAacson, D. MARChesin, B. Plohr, AND B. Temple, The Riemann problem near a hyperbolic singularity: The classification of solutions of quadratic Riemann problems 1, SIAM J. Appl. Math., 48 (1988), pp. 1009-1032.

[6] E. ISAACSON AND B. TEMPLE, The structure of asymptotic states in a singular system of conservation laws, Adv. Appl. Math., 11 (1990), pp. 205-219.

[7] _- Analysis of a singular system of conservation laws, J. Differential Equations, 65 (1986), pp. 250268.

[8] - Examples and classification of non-strictly hyperbolic systems of conservation laws, Abstracts of Amer. Math. Soc., Providence, RI, January 1985.

[9] B. KEYFITZ AND H. KRANZER, A system of non-strictly hyperbolic conservation laws arising in elasticity theory, Arch. Rat. Mech. Anal., 72 (1980), pp. 219-241.

[10] P. D. LAX, Hyperbolic systems of conservation laws, II, Comm. Pure Appl. Math., 10 (1957), pp. 537-566.

[11] T. P. LIU, Quasilinear hyperbolic systems, Comm. Math. Phys., 68 (1979), pp. 141-172.

[12] - Resonance for a quasilinear hyperbolic equation. J. Math. Phys., 28 (1987).

[13] D. Marchesin AND P. J. PAes-Leme, A Riemann problem in gas dynamics with bifurcation, PUC Report MAT 02/84, 1984.

[14] O. A. Oleinik, Discontinuous solutions of non-linear differential equations, Uspekhi Mat. Nauk, 12 (1957), pp. 3-73; Amer. Math. Soc. Trans., Ser. 2, 26, pp. 195-172.

[15] D. SCHAEFFER AND M. SheARER, The classification of $2 \times 2$ systems of conservation laws, with application to oil recovery, with Appendix by D. Marchesin, P. J. Paes-Leme, D. Schaeffer, and M. Shearer, Comm. Pure Appl. Math., 40 (1987), pp. 141-178.

[16] J. SMoller, Shock Waves and Reaction Diffusion Equations, Springer-Verlag, Berlin, New York, 1983.

[17] B. TEMPLE, Global solution of the Cauchy problem for a class of $2 \times 2$ nonstrictly hyperbolic conservation laws, Adv. Appl. Math., 3 (1982), pp. 335-375. 